de las nuevas categorías UICN a la flora endémica de la Comunidad Valenciana. Bol. Real Soc. Españ. Hist. Nat. Tomo Extraord. 125 Anivers.: 385-387.

LETSCHERT, P. P. W. -1993-Beta section Beta: biogeographical patterns of variation and taxonomy. Wageningen Agric. Univ. Papers 93(1): 1-155.

MATEO, G. y M. B. CRESPO-1998-Manual para la determinación de la flora valenciana. Monogr. Fl. Montiberica ${ }^{\circ} 3$. Valencia.

RITA, J y G. BIBILONI -1993- La vegetación (Memòria del mapa de les comunitats vegetals). En: Alcover, J. A., E. Ballesteros y J. J. Fornós (eds.): Història natural de l'Arxipèlag de Cabrera. Consell Superior d'Investigacions Científiques. Ed. Moll. Mallorca.
RIVAS MARTÍNEZ, S. -1987-Memoria del mapa de series de vegetación de España 1:400.000. ICONA - Mº Agricultura, Pesca y Alimentación. Madrid.

Aceptado para su publicación en Octubre de 1999

Dirección de los autores. Dpto. Ciencias Ambientales y Recursos Naturales (Botánica). Universidad de Alicante. Apdo. 99. E-03080 Alicante. e-mail: crespo@carn.ua.es

\title{
84. SOBRE PRIMULA HIRSUTA ALL. Y LA NOMENCLATURA DE DOS ASOCIACIONES PIRENAICAS DEL ANDROSACION VANDELLII
}

\author{
José Luis BENITO ALONSO
}

About Primula hirsuta All. and the nomenclature of two pyrenean associations from Androsacion vandellii

Palabras clave. Fitosociología, nomenclatura, Primula hirsuta, Primula latifolia, Androsacion vandellii, España, Francia, Pirineo.

Key words. Phytosociology, nomenclature, Primula hirsuta, Primula latifolia, Androsacion vandellii, Spain, France, Pyrenees.

Primula hirsuta All. y Primula latifolia Lapeyr. son dos primuláceas que pertenecen a la misma sección (Aleurita Duby), por lo que muchos autores las han confundido. En pliego de herbario, $P$. hirsuta se distingue por tener pelos glandulíferos con la célula terminal de color rojizo, mientras que $P$. latifolia los tiene de color pálido, amarillento. En fresco, el color

Trabajo realizado con la financiación del Departamento de Educación y Cultura del Gobierno de Aragón, a través de una beca predoctoral de investigación. 
de la flor de esta última es violáceo-rojizo, no más clara en la garganta, aunque algo farinosa, y con el escapo generalmente más largo que las hojas, e inflorescencia \pm unilateral. Por el contrario la planta de Allioni es de color rosa lila y caracteres antitéticos a los descritos para P. latifolia (Vargas, 1997: 11).

La distribución de ambas es alpinopirenaica, aunque en nuestra cadena se comportan como vicariantes a uno y otro extremo de la misma, pues viven en los mismos ambientes y altitudes. Nunca llegan a encontrarse, pues dejan un hueco que va de la zona oriental del Pirineo central a la parte occidental del Pirineo oriental. Puede verse un mapa actualizado y localidades detalladas en Benito Alonso (1999).

En el Pirineo, la distribución de Primula hirsuta es centro-occidental (Villar et al., 1999). En la vertiente española la encontramos en Huesca (Aragón), desde el puerto de Somport (valle del Aragón) por el oeste hasta el macizo de Posets (valle del Ésera) por el este. En la parte francesa alcanza el pico del Anie (Béarn, Pirineos Atlánticos), aunque es más abundante en la zona central de la cadena, llegando hasta el puerto de Oô y el pico de Gar en el Alto Garona.

Por otra parte, Primula latifolia es planta que en el Pirineo tiene una distribución oriental: se localiza en Gerona (Cataluña), en el aito Ripollés, llegando a penetrar en la Cerdaña. En la parte francesa se reparte principalmente por el departamento de los Pirineos Orientales, tanto en el Vallespir por la cuenca alta del río Tech, como en el Conflent por la cuenca alta del río Tet, entrando puntualmente en el alto Ariège y llegando casi hasta Andorra.

Ambas Primulae viven preferentemente en las grietas de los roquedos silíceos de los pisos subalpino al subnival, formando parte de comunidades de la alianza Androsacion vandellii. Br.-B1. in Br.-B1. \& Jenny 1926.

Saxifrago cotyledonis-Primuletum hirsutae
Fern. Casas 1970 corr. Benito Alonso 1999 [Holotypus: Fernández Casas, Ars. Pharm. 11: 276, inv. único. 1970]

Syn.: Saxifrago (cotyledonis)-Primuletum viscosae Fern. Casas 1970, op. cit.: 276, nom. incorr. (Art. 43); Hyperico nummulariSaxifragetum cotyledonis Rivas Mart., Báscones, T.E. Díaz, Fern. Gonz. \& Loidi 1991, Itinera Geobot. 5: 388, nom superfl.

Discusión. Fernández Casas (1970), al describir de Panticosa el Saxifrago (cotyledonis)-Primuletum viscosae toma como especie característica Primula viscosa All., que es sinónimo de $P$. latifolia, planta pirenaico oriental que no está en el citado valle panticuto, por lo que se produce un error taxonómico en la descripción de dicha comunidad. En virtud del artículo 43 del Código de Nomenclatura Fitosociológica (Barkman et al., 1988) hay que corregir el nombre y cambiar el epíteto viscosae por hirsutae.

Los dos inventarios que aportan Rivas Martínez et al. (1991: 389, invs. 5 y 6) para la descripción de su Hyperico nummulariiSaxifragetum cotyledonis son sustancialmente iguales al que sirve para caracterizar la asociación de Fernández Casas (op. cit.), por lo que la sinonimizamos a esta última.

Pensamos, al igual que Fernández Casas (op. cit.), que esta asociación debe estar incluida en la alianza Androsacion vandelli, pues aunque muy pobre en especies de la misma y del orden, no posee ninguna especie característica de la alianza Violo bifloraeCystoteridion alpinae Fern. Casas 1970 (=Cystopteridion J.L. Richard 1972) que es donde la encuadran Rivas Martínez et al. (op. cit.: 386). Debemos tener en cuenta que algunas de las especies que dichos autores incluyen en el Cystopteridion, no las consideramos dentro de dicha alianza. Así, compartimos la opinión de Bolòs \& Vigo (1984: 313) de que Saxifraga aizoides $\mathrm{L}$. es característica de asociaciones 
del Cratoneurion commutati W. Kock 1928, Saxifragion praetermissae Rivas. Mart. 1977, Caricion davallianae Klika 1934, etc. También creemos que Saxifraga cotyledon L. cuadra mejor en el Androsacion vandelli.

Ecología y táxones característicos. Comunidad de las grietas de las rocas silíceas aunque ricas en carbonatos, rezumantes 0 húmedas buena parte del año, de los pisos montano alto y subalpino. Es una asociación caracterizada por la presencia de Saxifraga cotyledon y Primula hirsuta, así como por especies del orden Androsacetalia vandellii como Poa nemoralis subsp. glauca y esporádicamente Androsace vandellii, y de la clase Asplenietea trichomanis como Saxifraga paniculata o Hypericum nummularium.

Distribución. Por el momento sólo se conoce del lugar de descripción, Panticosa (Pirineo central, Huesca), aunque podría encontrarse también en Benasque y en el área de distribución de Saxifraga cotyledon en el Pirineo.

\section{Cardamino resedifoliae-Primuletum hirsutae}

(Turmel 1955) Benito Alonso 1999, nom. nov.

[Lectotypus (designado aquí): Turmel, Mém. Mus. Hist. Nat., sér. B, Bot. 5: 87, tab. 16, inv. 6. 1955]

Syn.: Asplenio septentrionalis-Primuletum viscosae Turmel 1955, nom. incorr. et illeg.(Arts. 43 y 31); Asplenio septentrionalisPrimuletum latifoliae Rivas Mart. et al. 1991, op. cit., nom. incorr. et illeg. (Arts. 43 y 31); Asplenio septentrionalis-Primuletum hirsutae Rivas Mart. et al. 1991 corr. Villar, Sesé \& Ferrández 1997: LXXII, Atlas de la Flora Pirineo del Aragonés, nom. illeg. (Art. 31); non Asplenio septentrionalis-Primuletum hirsutae (Lüdi 1921) Br.-Bl. in Meier \& Br.Bl. 1934, Prodrome des groupements végétaux, fasc. 2. Groupements rupicoles

Discusión. Turmel (1955: 87), al describir el Asplenio septentrionalis-Primuletum viscosae del macizo del Midi d'Ossau (Pirineo occidental), toma como especie característica Primula viscosa All. (op. cit.: 74), que es sinónimo de $P$. latifolia Lapeyr., planta que, como ya hemos dicho, es pirenaico oriental y no se encuentra en la localidad de donde se describe dicha comunidad. Se ha producido, por tanto, un error taxonómico, y en virtud del artículo 43 del C.N.F. hay que corregir el nombre y cambiar el epíteto viscosae por hirsutae, cosa que hacen de facto Villar et al. (1997: LXXII). Sin embargo, no advierten que crean un nombre ilegítimo por homonimia posterior con el Asplenio septentrionalisPrimuletum hirsutae (Lüdi 1921) Br.-Bl. 1934 de los Alpes, por lo que debe rechazarse dicha corrección (art. 31) y proponerse la creación de un nombre nuevo (art. 39).

Rivas Martínez et al. (op. cit.: 388), describieron una asociación de los roquedos silíceos del Pirineo central tomando como especie característica Primula latifolia, y llamando a la comunidad Asplenio septentrionalis-Primuletum latifoliae. Sin embargo, como ya hemos comentado anteriormente, dicha prímula es pirenaico oriental, mientras que en las localidades que se dan en la tabla de inventarios $n^{\circ} 72$ (op. cit.: 389-390) lo que realmente aparece, según los testimonios depositados en el herbario JACA de esas mismas zonas, es sin lugar a dudas Primula hirsuta All. Además, según nuestra opinión, esta comunidad es la misma que describió Turmel en los años 50, por lo que la sinonimizamos a aquélla.

Ecología y táxones característicos. Comunidad de grietas de paredes tumbadas (< $90^{\circ}$ de inclinación), aunque a veces también en las verticales, de rocas graníticas y esquistosas de los pisos subalpino y alpino. Tiene como especies características a Primula hirsuta, Asplenium septentrionale, Silene rupestris, Sempervivum montanum; a veces Androsace pyrenaica, Saxifraga iratiana, S. pentadactylis 
y baja presencia de Androsace vandellii (sólo en los valles de Tena y Benasque).

Distribución. Se distribuye por el Pirineo central básicamente, aunque también alcanza el occidental: en Huesca desde la cabecera del río Aragón (Somport) hasta el valle de Benasque, y por Francia desde el pico del Anie (Pirineos Atlánticos) hasta el valle de Aure (Altos Pirineos).

La nomenclatura de las subasociaciones definidas Rivas Martínez et al. (op. cit.), queda de la siguiente forma:

\section{Cardamino resedifoliae-Primuletum hirsutae}

(Turmel 1955) Benito Alonso 1999 primuletosum hirsutae, nom. nov.

[Lectotypus (designado aquí): Turmel, op. cit: 87 , tab. 16, inv. 6. 1955]

Syn.: Asplenio septentrionalis-Primuletum latifoliae Rivas Mart. et al. 1991 primuletosum latifoliae Rivas Mart. et al. 1991, nom. incorr. (Art. 43)

Según Rivas Martínez et al. (op. cit: 388) la subasociación típica sería principalmente subalpina y de rocas silíceas pobres en bases, y por tanto faltarían plantas calcícolas.

\section{Cardamino resedifoliae-Primuletum hirsutae}

(Turmel 1955) Benito Alonso 1999 saxifragetosum iratianae (Rivas Mart. et al. 1991) Benito Alonso 1999, comb. nov. [Holotypus: Rivas Martínez et al., op. cit.: 390, tab. 72, inv. 8]

Bas.: Asplenio septentrionalis-Primuletum latifoliae Rivas Mart. et al. 1991 saxifragetosum iratianae Rivas Mart. et al. 1991, nom. incorr. (Art. 43)

Según Rivas Martínez et al. (op. cit: 388), viviría en los pisos subalpino superior y alpino, con Saxifraga iratiana y Potentilla nivalis como especies diferenciales.

Cardamino resedifoliae-Primuletum hirsutae (Turmel 1955) Benito Alonso 1999 potentilletosum alchimilloidis (Rivas Mart. et al. 1991) Benito Alonso 1999, comb. nov. [Holotypus: Rivas Mart. et al., op. cit.: 390, tab. 72, inv. 12]

Bas.: Asplenio septentrionalis-Primuletum latifoliae Rivas Mart. et al. 1991 potentilletosum alchimilloidis Rivas Mart. et al. 1991, nom. incorr. (Art. 43)

Según Rivas Martínez et al. (op. cit: 388), esta subasociación tendría una distribución subalpina y alpina inferior, sobre rocas silíceas ricas en bases, con algunos casmófitos calcícolas diferenciales como Potentilla alchimilloides, Globularia repens, Lonicera pyrenaica y Asplenium ruta-muraria.

AGRADECIEMIENTOS: A los Drs. M.B. Crespo y F. Fernández González por la revisión crítica del manuscrito.

\section{BIBLIOGRAFÍA}

BARKMAN, J.J., J. MORAVEC \& S. RAUSCHERT -1988-Código de nomenclatura fitosociológica. Opus. Bot. Pharm. Complutensis 4: 9-74.

BENITO ALONSO, J.L. -1999- Cartografía corológica ibérica. Mapas de Primula hirsuta All. y P. latifolia Lapeyr. Bot. Complutensis 23. En prensa.

BOLÒS, O. \& J. VIGO -1984- Flora dels Països Catalans, vol. 1. Introducció. (LycopodiaceaeCapparaceae). Editorial Barcino. 736 pp. Barcelona.

FERNÁNDEZ CASAS, J. -1970- Notas fitosociológicas breves, I. Ars. Pharm. 11: 273298.

MEIER, H. \& J. BRAUN-BLANQUET -1934Prodrome des groupements végétaux, fasc. 2. Groupements rupicoles. Imprimerie Mari-Lant. Montpellier.

RIVAS MARTÍNEZ, S., J.C. BÁSCONES, T.E. DÍAZ, F. FERNÁNDEZ GONZÁLEZ y J. LOIDI -1991- Vegetación del Pirineo occidental y Navarra. Itinera Geobot. 5: 5-456.

TURMEL, J.-M. -1955- Le pic du Midi d'Ossau. Ecologie et végétation. Mém. Mus. Hist. Nat., 
sér. B, Bot. 5: 1-208.

VARGAS, P. -1997- IN CASTROVIEJO, S., C. Aedo, M. Laínz, R. Morales, F. Muñoz Garmendia, G. Nieto y J. Paiva (eds.) Flora Iberica, vol. 5. Real Jardín Botánico, C.S.I.C. Madrid.

VILLAR, L., J.A. SESÉ y J.V. FERRÁNDEZ 1997-Atlas de la Flora del Pirineo Aragonés, I (Introducción. Lycopodiaceae-Umbelliferae). Consejo de Protección de la Naturaleza (D.G.A.) e Instituto de Estudios Altoaragoneses. XCI+ 648 pp. Huesca.
VILLAR, L., J.A. SESÉ y J.V. FERRÁNDEZ 1999-Atlas de la Flora del Pirineo Aragonés, II (Pyrolaceaee-Orchidaceae). Consejo de Protección de la Naturaleza (D.G.A.) e Instituto de Estudios Altoaragoneses. Huesca. En prensa.

Aceptado para su publicación en septiembre de 1999

Dirección del autor. Instituto Pirenaico de Ecología, C.S.I.C. Apdo. 64. E-22700 Jaca. Huesca. España. Correo-e.: jolube@server-j1.ipe.csic.es 